Scientific Journal of Hamadan Nursing \& Midwifery Faculty - ISSN 2008-2819

\title{
Effect of Group Counseling on the Type of Delivery in Nulliparous Women: A Randomized Controlled Trial
}

\author{
Zinat Jourabchi ${ }^{1}$, Zahra Roshan ${ }^{2}$, Mahmod Alipour-Heidari ${ }^{3}$, Fatemeh Ranjkesh ${ }^{*}$ \\ 1. Assistant Professor, Department of Midwifery, Faculty of Nursing and Midwifery, Qazvin University of Medical \\ and Health Sciences, Qazvin, Iran \\ 2. Student of Midwifery Counseling, Faculty of Nursing and Midwifery, Qazvin University of Medical and Health \\ Sciences, Qazvin, Iran \\ 3. Assistant Professor, Department of Biostatistics, School of Medical Sciences, Qazvin University of Medical \\ Sciences, Qazvin, Iran \\ 4. Insctructor, Department of Midwifery, School of Nursing and Midwifery, Qazvin University of Medical Sciences, \\ Qazvin, Iran

\begin{tabular}{ll}
\hline Article Info & Abstract \\
\hline
\end{tabular}

Received: $\quad 2017 / 09 / 26$

Accepted: $\quad$ 2018/03/16

Published Online 2018/03/16

DOI: $10.30699 /$ sjhnmf.26.2.120

Original Article

Use your device to scan and read the article online

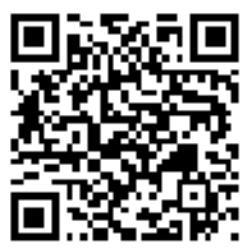

Background: Pregnancy and childbirth is one of the most important stages in women's lives. One of the reasons for women's desire to choose interest in cesarean delivery is lack of enough knowledge in choosing their birth method. This research studies the effect of group counseling on the type of delivery in Nulliparous women.

Methods: This is a controlled randomized trial will be conducted in 160 pregnant nulliparous women referred to Health Center from 95- 96 in Hamedan. The inclusion criteria were as follows: Women with gestational age 20-23 weeks. The exclusion criteria were as follows: maternal medical and Psychological diseases. The samples were randomly allocated to group intervention $(\mathrm{n}=80)$ and control groups $(\mathrm{n}=80)$. In both groups, mothers received routine prenatal care. The intervention group additionally received 8 sessions weekly group counseling. Data was collected by a checklist consisting of demographic, pregnancy, and delivery information and was analyzed by statistical testes such as T-test and chi squares. $P<0.05$ was considered as significant.

Result: A significant difference was also observed between two groups in the rate of normal vaginal delivery $(P=0.04)$. The results indicated that the group counseling significantly increased the physiologic delivery (53.2\%) in the intervention group compared by the control group $(22 / 2 \%)(P=0 / 002)$.

Conclusion: The results of the study showed that the presence of nulliparous women in the group counseling increases the rate of physiologic delivery and is suggested as one of the methods of implementing pregnant women's care program.

Keywords: Counseling, Nulliparous Women, Pregnancy, Childbirth

Fatemeh Ranjkesh, Insctructor, Department of Midwifery, School of Nursing and Midwifery, Qazvin University of Medical Sciences, Qazvin, Iran

E-mail: fatemehranjkesh@yahoo.com, Tel: 028-13359501

Copyright (C) 2018, Sci J Hamadan Nurs Midwifery Fac. This is an open-access article distributed under the terms of the Creative Commons Attribution-noncommercial 4.0 International License which permits copy and redistribute the material just in noncommercial usages, provided the original work is properly cited.

How to Cite This Article:

Jourabchi Z, Roshan Z, Alipour M, Ranjkesh F. Effect of Group Counseling on the Type of Delivery in Nulliparous Women: A Randomized Controlled Trial. Sci J Hamadan Nurs Midwifery Fac. 2018; 26 (2): $120-128$ 


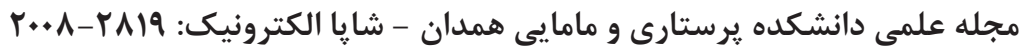

مقاله يثوهشى

تأثير مشاورة كروهى بر نوع زايمان مادران نخستزا: يك كار آزمايى بالينى

زينت جورابجى'، زهرا روشن"، محمود عليِور حيدرى"، فاطمه رنجكش"

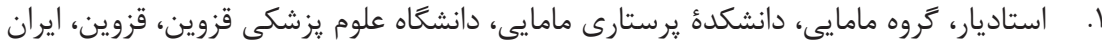

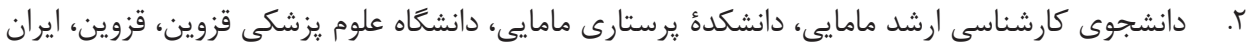

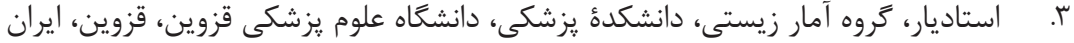

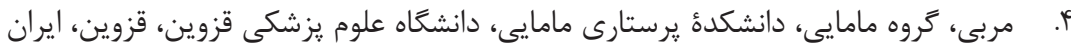

\begin{tabular}{|c|c|}
\hline جكيده & 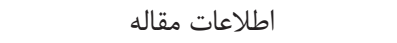 \\
\hline 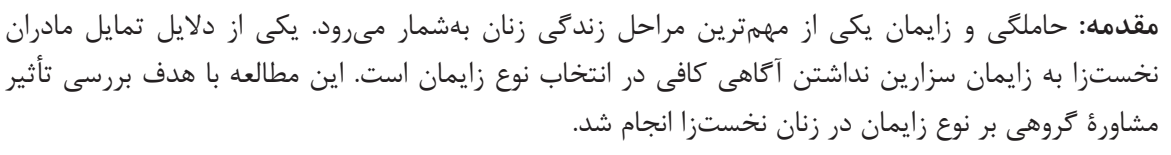 & $\begin{array}{ll}\text { تاريخ وصذئ: } & \text { تاريخ: }\end{array}$ \\
\hline 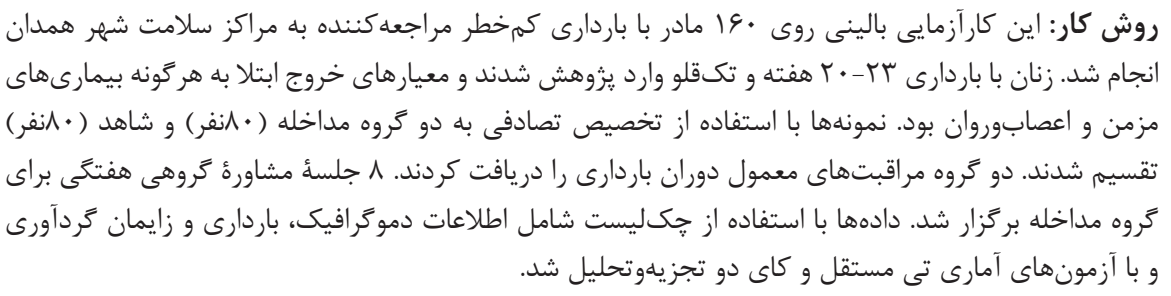 & 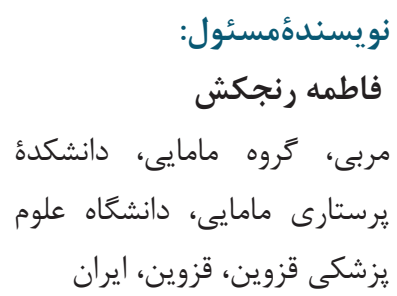 \\
\hline 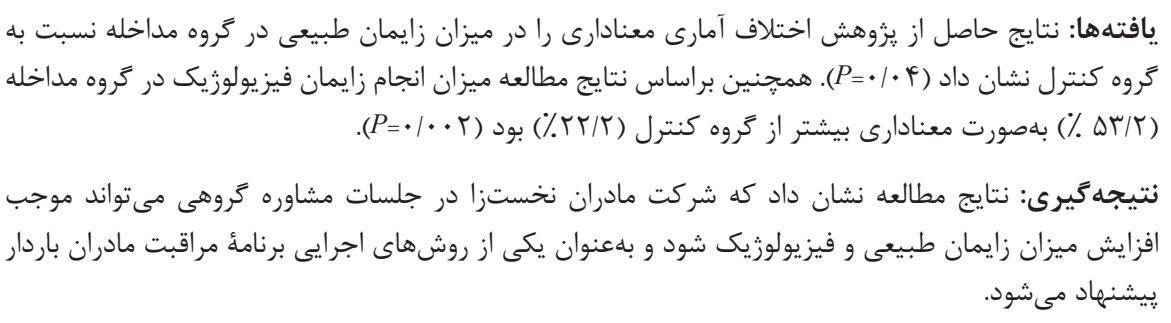 & 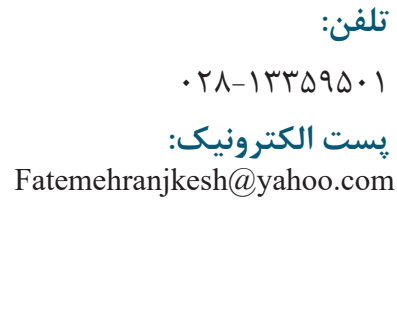 \\
\hline كلمات كليدى: زنان نخستزا، مشاوره، زايمان، باردارى & \\
\hline
\end{tabular}

زايمان طبيعى در مقايسه با سزارين مزاياى بسيارى دارد؛

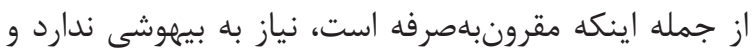

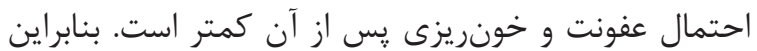

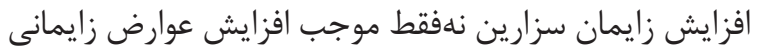

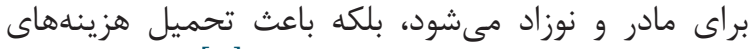

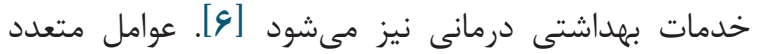

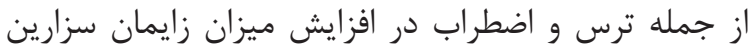

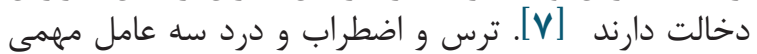

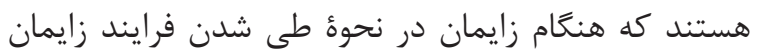

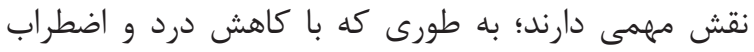

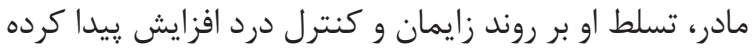

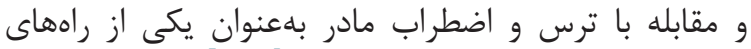

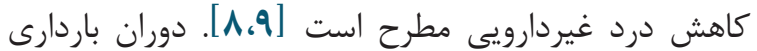

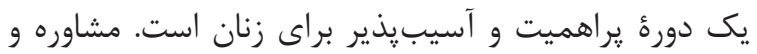

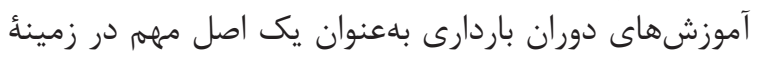

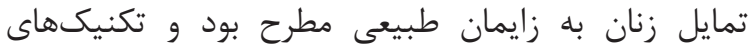

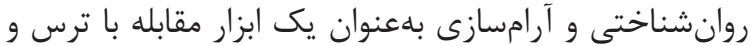

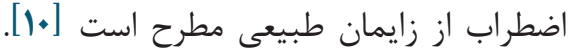

زايمان و تولد انسان يكى از لحظات مقدس و باشكوه

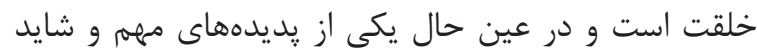

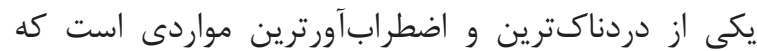

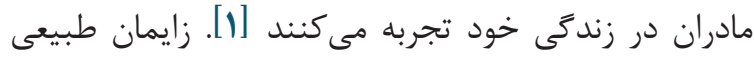

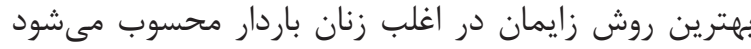

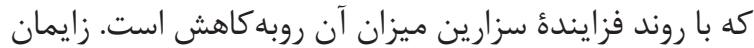

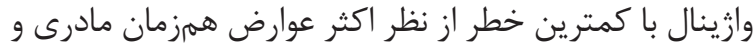
جنينى همراه است [باست

سزارين يكى ازشايعترين اعمال جراحى در زنان است.

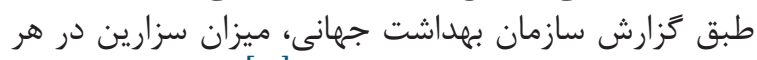

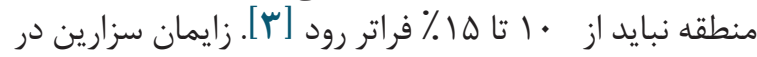

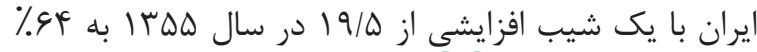

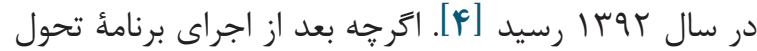

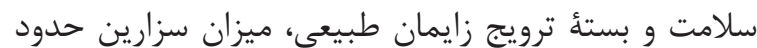

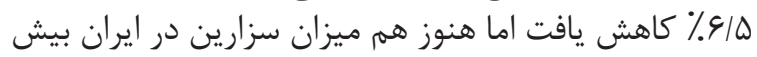

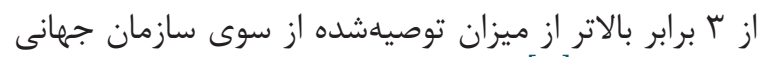

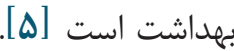


معيارهاى ورود به مطالعه شامل زنانى مى شعد كه در هفتأ

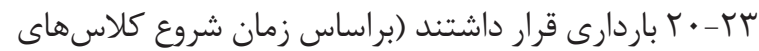

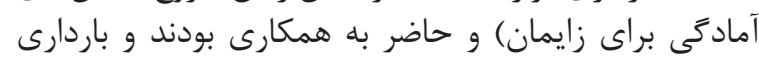

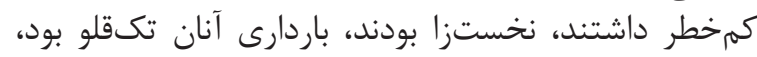

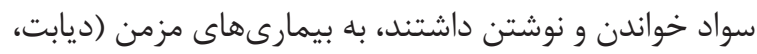

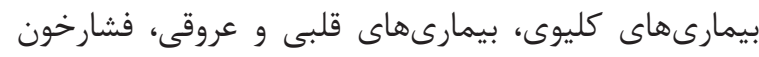

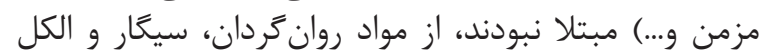

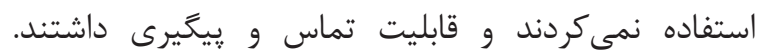

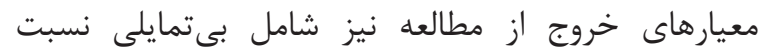

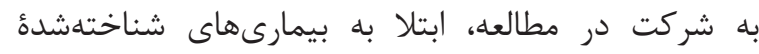

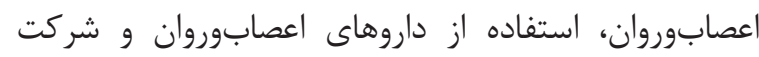

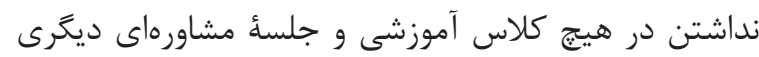

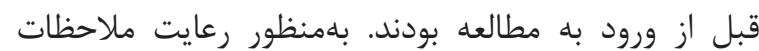

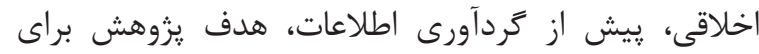

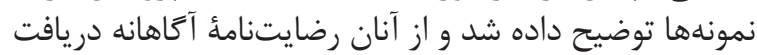

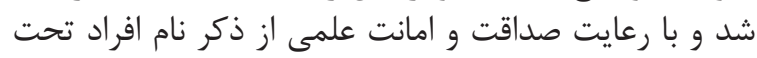

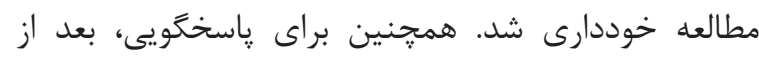

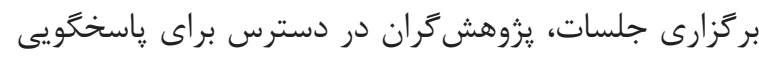

بودند.

نمونهها قبل از ورود به يزوهش بارئ تكميل خكليست

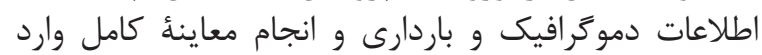

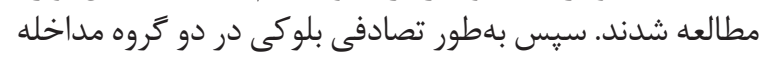

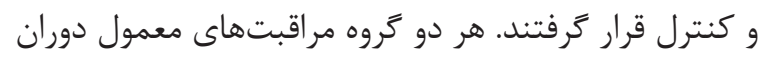

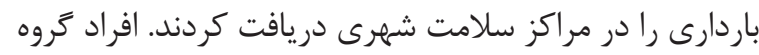

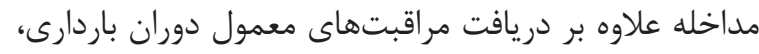

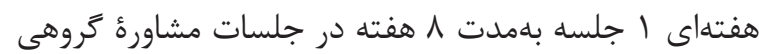

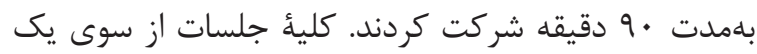

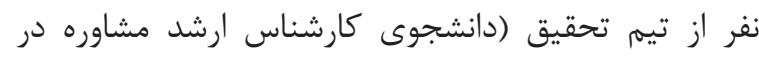

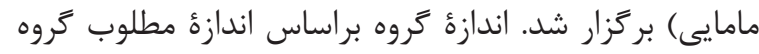

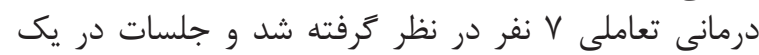

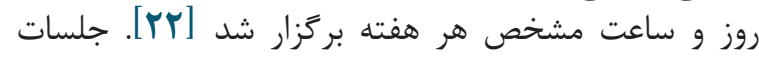

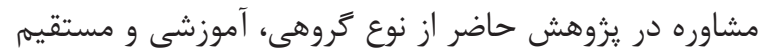

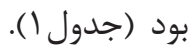

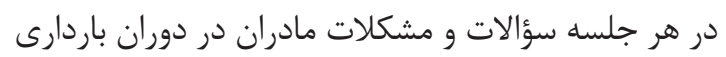

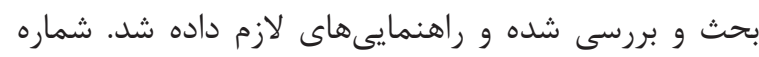

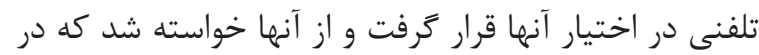

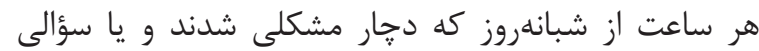
داشتند، با مشاور تماس بخيرند.

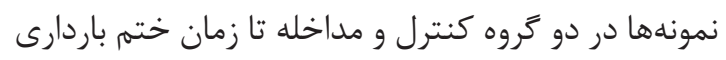

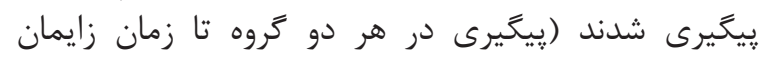

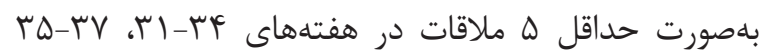

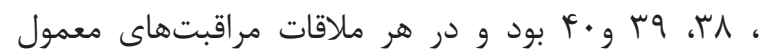

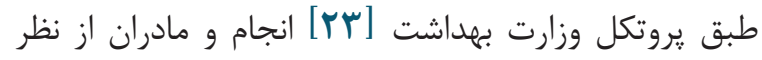

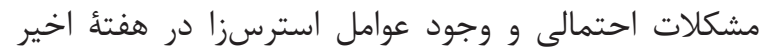

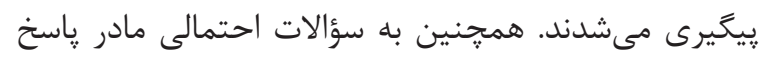

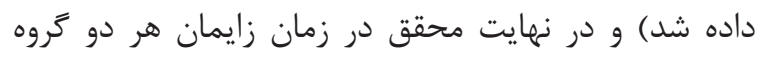

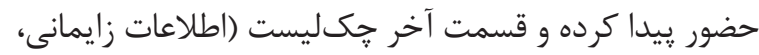

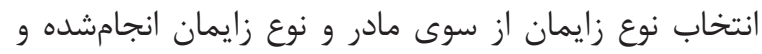

مشاورة سلامت يك فرايند روانشناختى ــ آموزشى است

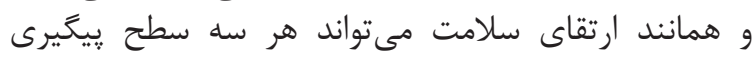

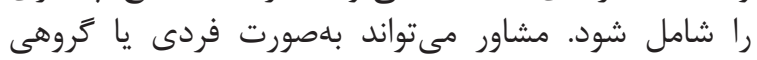

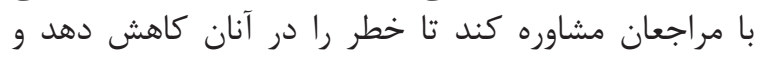

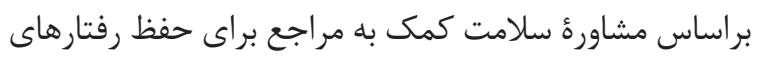

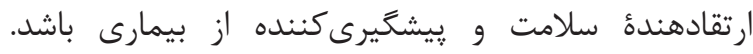

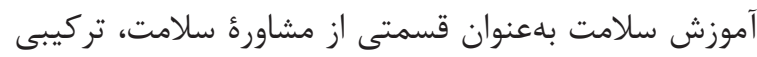

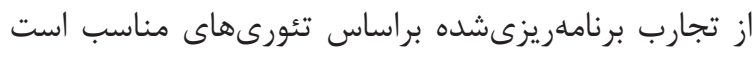

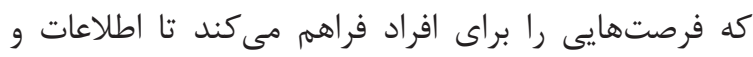

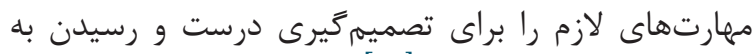

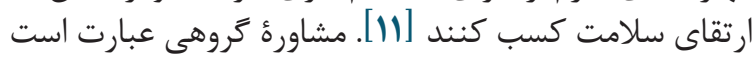

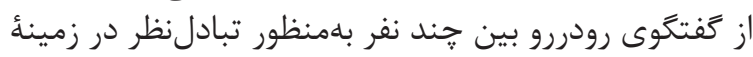

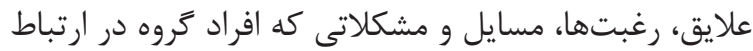

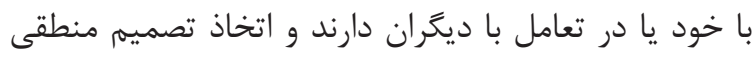

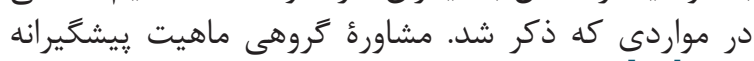

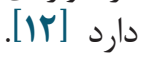

Nosratabadi

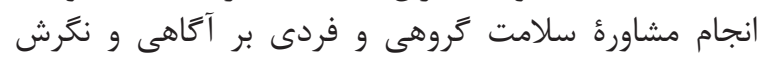

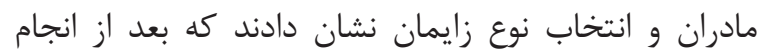

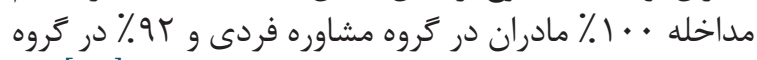

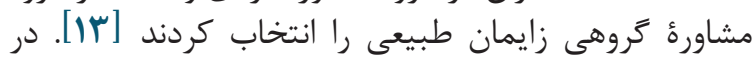

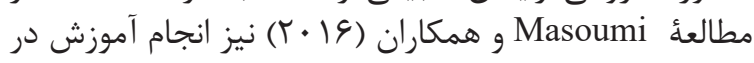

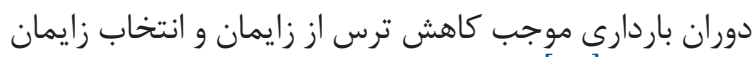

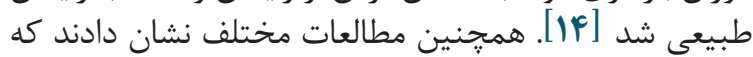

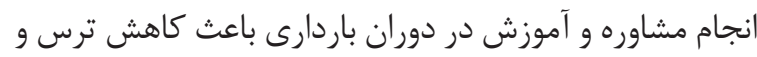

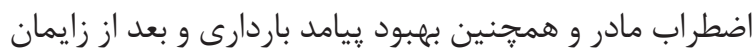

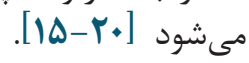

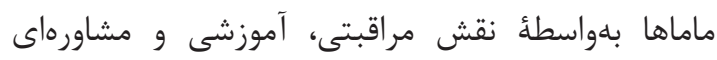

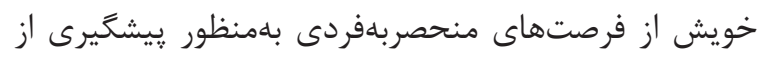

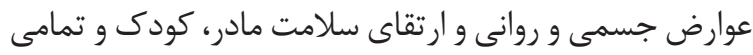

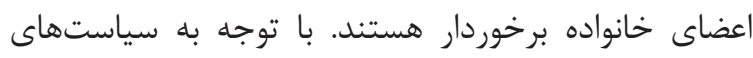

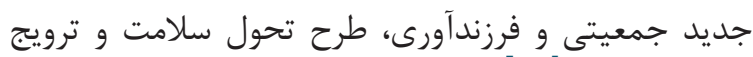

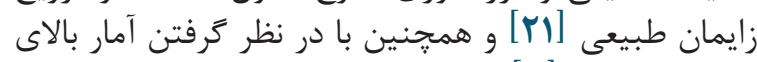

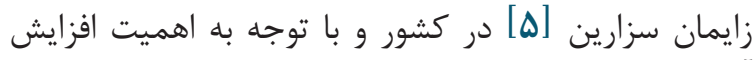

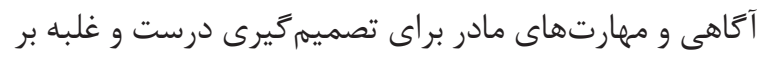

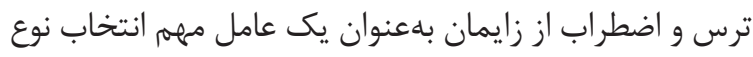

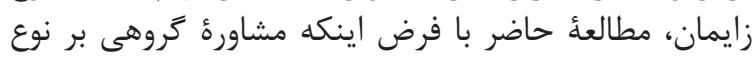

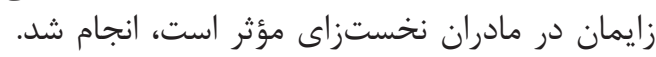

\section{روش كار}

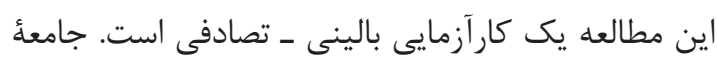

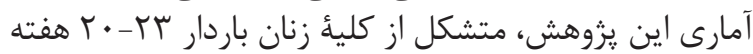

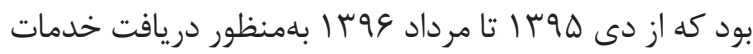

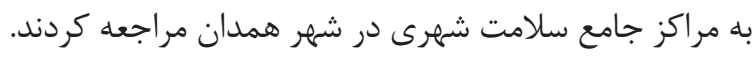

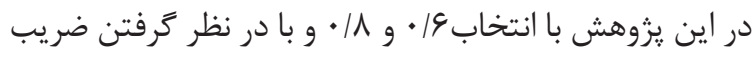

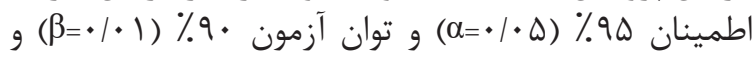

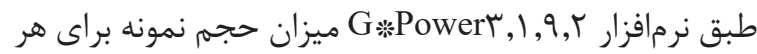

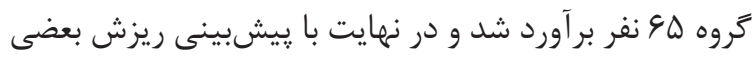

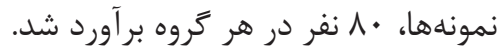




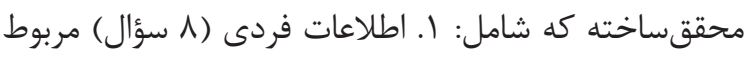

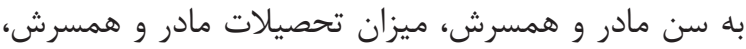

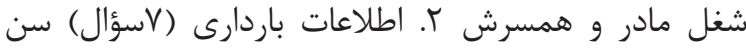

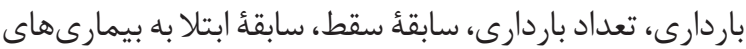

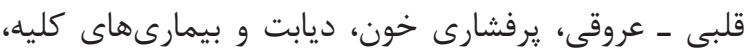

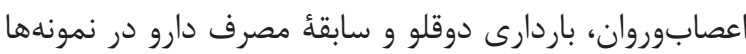

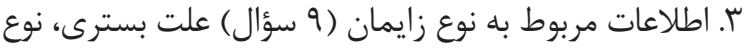

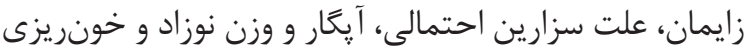

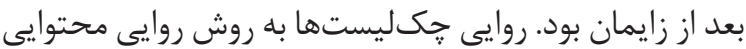

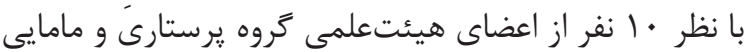

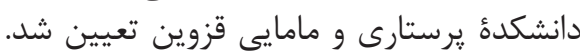

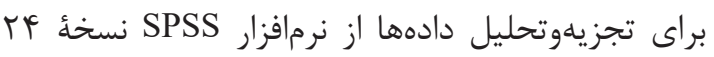

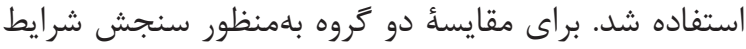

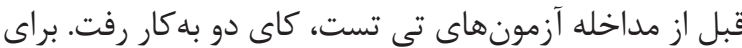

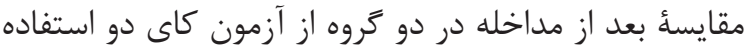

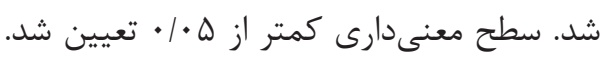

علت سزارينهاى احتمالى) را تكميل كرد.

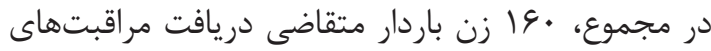

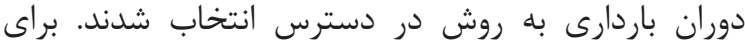

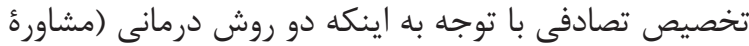

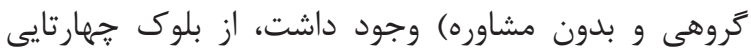

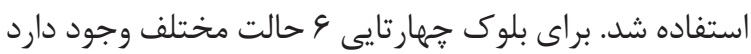
(AAaa,aaAA,AaAa.aAaA,AaaA,aAAa)

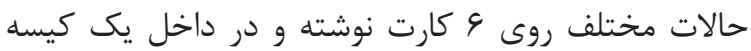

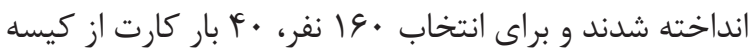

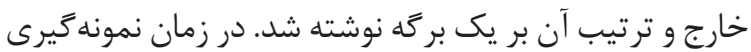

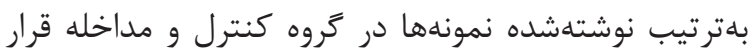

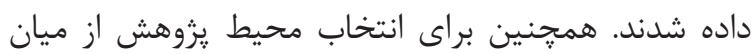

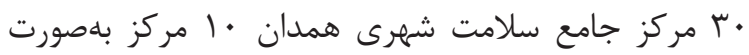
تصادفى از روى جدول اعداد تصادفى انتخاب شدند ابزار استفادهده در اين مطالعه شامل: جكىليست

جدول شماره ا. مباحث مطرحشده در جلسات مشاوره

آشنايى با اعضاى كروه، بيان اهداف جلسات مشاوره، فراهم آوردن شرايط براى ايجاد روابط و ارتباط باز، تأكيد

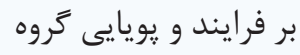

جلسئ اول

آشنايى با فيزيولوزى باردارى، تغييرات فيزيولوزئيك دورئ دوران باردارى، آموزش اصلاح وضعيت، تمرينات تنفسى،

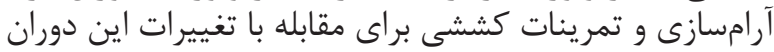

جلسئ دوم

آشنايى با بهداشت روان، يذيرش نقش والدى، نحوه ارتباط با جنين، اصلاح وضعيت در باردارى، انجام تمر ينات

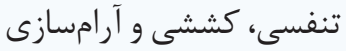

جلسةٔ سوم

آشنايى با زايمان و فرايند و مراحل آن، انواع روشهاى زايمان (زايمان طبيعى و سزارين) و مزايا و معايب آنها،

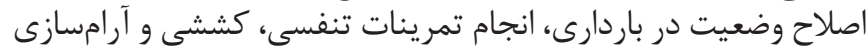

جلسئ جهارم

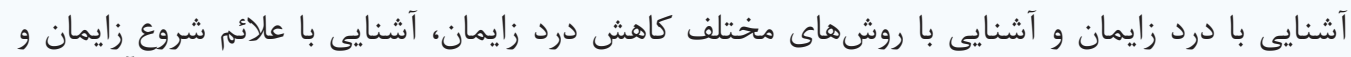

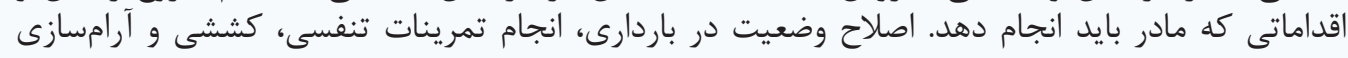
جلسهُ هنجم

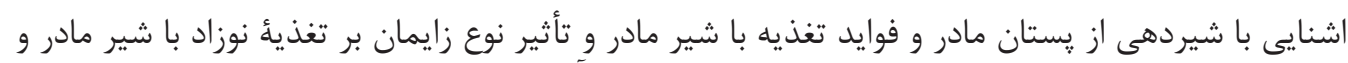

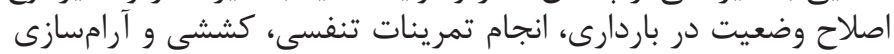

دوره بعد از زايمان و مراقبتهاى اين دوران در دو نوع زايمان طبيعى و سزارين، اصلاح وضعيت در باردارى، انجام تمرينات تنفسى، كششى و آرامسازى آنى دران دران

جلسةٔ هفتم آشنايى با دوران نوزادى، مراقبت از نوزاد، علائم خطر نوزاد و اصلاح وضعيت در باردارى، انجام تمرينات تنفسى، كششى و آرامسازى دوانى 


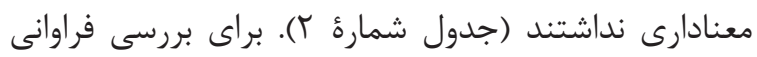

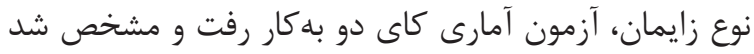

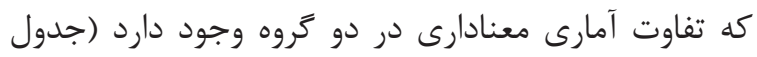

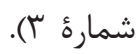

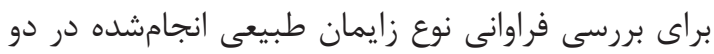

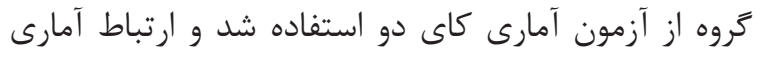

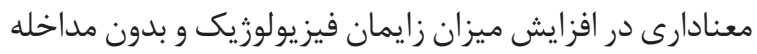

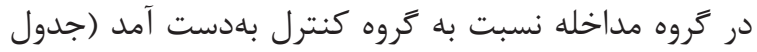

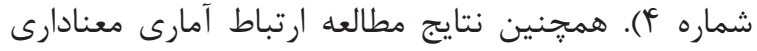

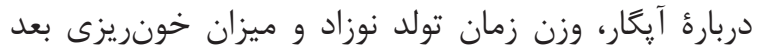

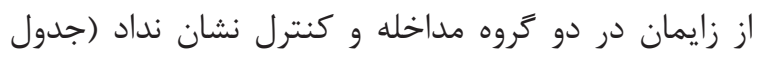

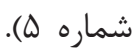

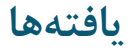

إئ مادر باردار ارزيابى اوليه شدند كه از اين تعداد

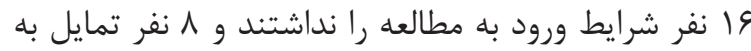

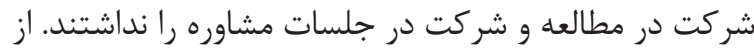

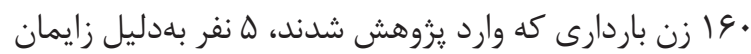

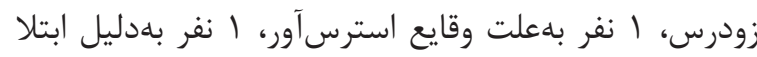

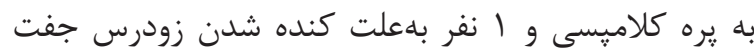

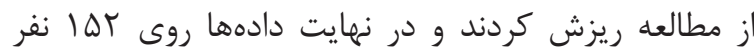
تجزيهوتحليل آمارى شدند ( نمودار (1).

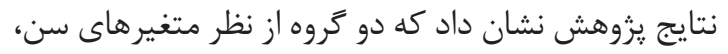

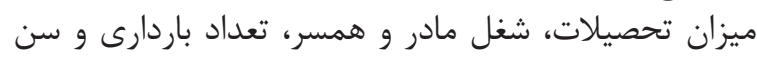

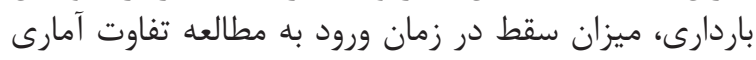

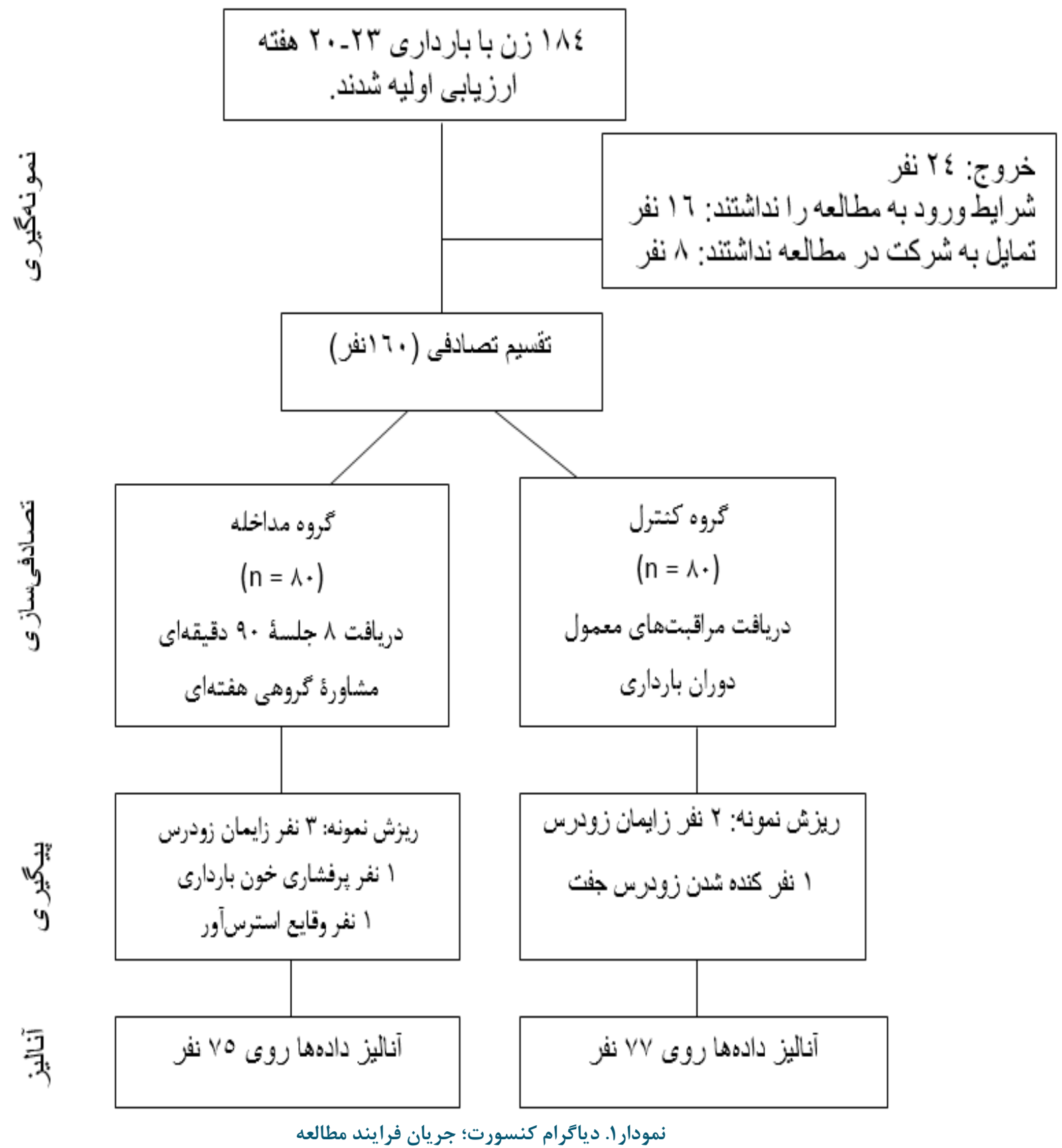


جدول شماره r. مقايسةٔ مشخصات جمعيتشناختى و باردارى در دو كَروه مداخله و كنترل

\begin{tabular}{|c|c|c|c|c|}
\hline$P$ value & $\underset{(n=V V)}{\text { كنترل }}$ & $\begin{array}{c}\text { مداخله } \\
(n=V \Delta)\end{array}$ & تروهها & متغير ها \\
\hline . KGY\% & $r V / r \Delta \pm \Psi / \Gamma$ & $r V / q V \pm r / 4 r$ & & سن مادر (سال) \\
\hline$\cdot / \pi \cdot 9 *$ & $r|/| r \pm r / \Delta \mid$ & $\Gamma / / q T \pm \Delta / / T$ & & سن همسر (سال) \\
\hline$\cdot \mid 9 \Delta \Lambda \%$ & KI/DSE./VIG & $T I / Q I \pm \cdot / V Y Y$ & & سن باردارى (هفته) \\
\hline \multirow{5}{*}{$\cdot / \Gamma ৭ \Delta * * *$} & $9(\%) 1 / \vee)$ & $10(\% \cdot r \cdot)$ & ابتدايى & \multirow{5}{*}{ ن تحصيلات خانم } \\
\hline & $1 \%(\% 19 / 9)$ & $10(\% \cdot r \cdot)$ & راهنمايى & \\
\hline & $r q(\% r V / V)$ & $r(\% r \Lambda)$ & متوسطه & \\
\hline & $r \xi(\%$ KT/A) & TF(\%T) & دانشخاهى & \\
\hline & $V(\% / 9)$ & $\mid r(\% V / \Gamma)$ & ابتدايى & \\
\hline \multirow[t]{5}{*}{$\cdot / r \Delta V_{* * *}$} & $r \mid(\% r V / 9)$ & $\mid \digamma(\% \mid \Lambda / V)$ & راهنمايى & \multirow{3}{*}{ تحصيلات همسر } \\
\hline & $r q(\% \Gamma N / r)$ & $r \Lambda(\% \Gamma / \Gamma)$ & متوسطه & \\
\hline & $19(\% / \Delta)$ & $r \cdot(\%$ Y $/ V)$ & دانشخاهى & \\
\hline & $(\% \wedge \vee) \& \vee$ & $(\% \wedge q / \uparrow) \& V$ & خانهدار & \multirow{3}{*}{ شغل خانم } \\
\hline & $(\% \vee / \Lambda) \&$ & $(\% \Delta / r))^{q}$ & 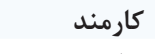 & \\
\hline \multirow[t]{3}{*}{$\cdot \mid \Lambda \Gamma \cdot * * *$} & $(\% \Delta / r)^{\varphi}$ & $(\% \Delta / \Gamma) \varphi$ & ساير & \\
\hline & $(\% r \Delta / r) 19$ & $(\% \cdot \cdot \mid \Lambda) \mid 9$ & كارمند & \multirow{4}{*}{ شغل همسر } \\
\hline & $(\% \mid N / V) \mid f$ & $(\% R r / F) \backslash \Lambda$ & كارتر & \\
\hline \multirow[t]{3}{*}{$\cdot / \vee \wedge \varepsilon_{* * *}$} & $(\% \Delta F / F) F \mid$ & $(\% \Delta r / r) F$ & آزاد & \\
\hline & $(\% 1 / \Gamma) 1$ & $(\% / 9) Y$ & 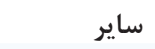 & \\
\hline & $(\% \mid Q / 9) \mid T$ & $(\% r \Delta / r) \mid q$ & دارد & \multirow{2}{*}{ سابقهُ سقط } \\
\hline \multirow[t]{2}{*}{ •/ノイ } & $(\% \wedge F / \uparrow) \& \Delta$ & $(\% V F / V) \Delta \varphi$ & 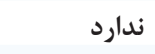 & \\
\hline & & & \multicolumn{2}{|c|}{ آزمون: :*تى تست ، ***كاى دو } \\
\hline
\end{tabular}

جدول شماره س. مقايسُٔ فراوانى نوع زايمان انجامشده در دو كروه كنترل و مداخله بعد از انجام مداخله

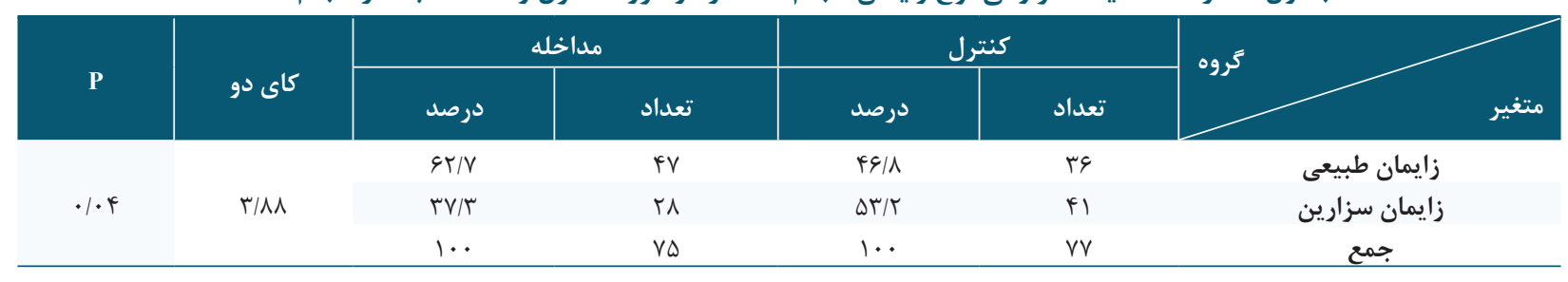

جدول شماره F. مقايسة فراوانى نوع زايمان طبيعى انجامشده در دو كَروه كنترل و مداخله بعد از انجام مداخله

\begin{tabular}{|c|c|c|c|c|c|c|}
\hline \multirow{2}{*}{$\mathbf{P}$} & \multirow{2}{*}{ 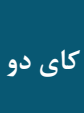 } & \multicolumn{2}{|c|}{ مداخله } & \multicolumn{2}{|c|}{ كنترل } & \multirow{2}{*}{ تروه } \\
\hline & & در ل د & تعداد & درصد & تعداد & \\
\hline \multirow{4}{*}{. $/ . r$} & \multirow{4}{*}{$\mid f / \Delta \Lambda$} & $r \Delta / \Delta$ & ir & $99 / V$ & TF & زايمان با مداخله \\
\hline & & $\Delta r / r$ & TQ & Tr/T & $\wedge$ & زايمان فيزيولوزيك \\
\hline & & $r \mid / r$ & 1. & $11 / 1$ & f & زايمان بىدرد \\
\hline & & $1 \ldots$ & FV & $1 \ldots$ & re & جمع \\
\hline
\end{tabular}

جدول شمارهُ ه. مقايسةُ يِيامد باردارى در دو تروه كنترل و مداخله بعد از انجام مداخله

\begin{tabular}{|c|c|c|c|c|c|c|c|}
\hline \multirow{2}{*}{$\mathbf{P}$} & \multirow{2}{*}{ كاى دو } & \multicolumn{2}{|c|}{ مداخله } & \multicolumn{2}{|c|}{ كنترل } & \multirow[t]{2}{*}{ 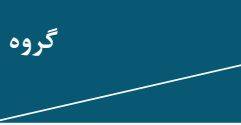 } & \multirow[b]{2}{*}{ متغير } \\
\hline & & درصد & تعداد & درصد & تعداد & & \\
\hline \multirow{3}{*}{./TrG } & \multirow{3}{*}{$\cdot / 1$} & $\Lambda \& / V$ & 90 & $\Lambda \cdot / \theta$ & GT & $r \Delta \cdots-r \ldots$ & \multirow{3}{*}{ وزن نوزاد (كرم) } \\
\hline & & $1 \pi / r$ & 1. & $1 \cdot / 4$ & $\wedge$ & كمتر از •." r & \\
\hline & & . & . & १/1 & v & بيشتر از ... F & \\
\hline \multirow{3}{*}{$\cdot \mid \Delta \wedge \Delta$} & \multirow{3}{*}{$r / V \Delta$} & 99 & VT & 1. & VV & $\wedge-1$. & \multirow{3}{*}{ آيحار دقيقئ ه } \\
\hline & & f & $r$ & . & . & $f-V$ & \\
\hline & & . & . & . & . & كمتر از F & \\
\hline \multirow{3}{*}{.$|91|$} & \multirow{3}{*}{$\cdot / r \Delta q$} & $\wedge$ & 9 & $1 \cdot / 4$ & $\wedge$ & دارد & \multirow{3}{*}{ خونريزى بعد از } \\
\hline & & 95 & 99 & $19 / 9$ & 99 & ندارد & \\
\hline & & $1 \ldots$ & VD & $1 \ldots$ & VV & & \\
\hline
\end{tabular}


كمك به مادر براى شناخت علت ترس و غلبه بر آن آنتخاب إنباب

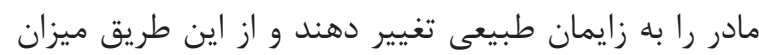

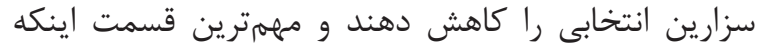

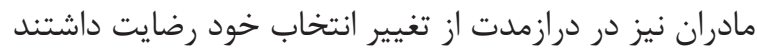

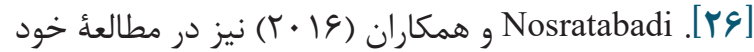

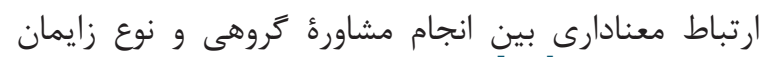

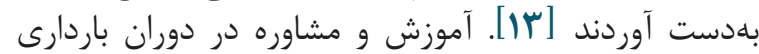

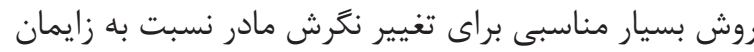

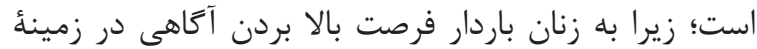

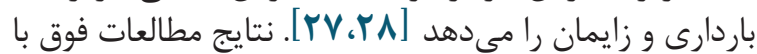
نتيجة مطالعهُ حاضر همسو است.

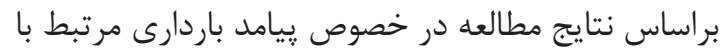

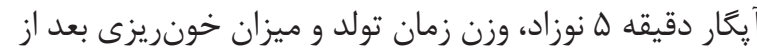

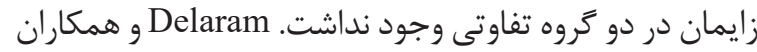

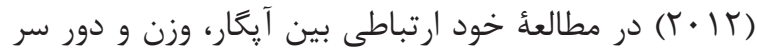

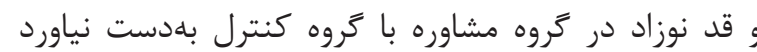

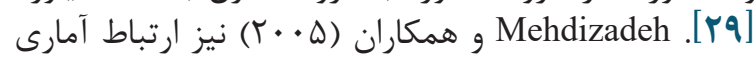

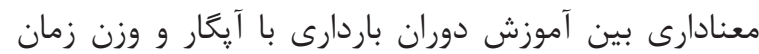

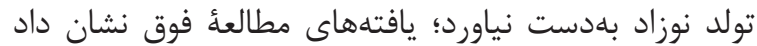

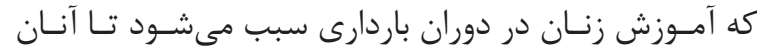

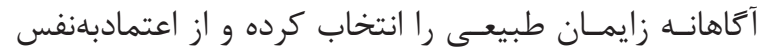

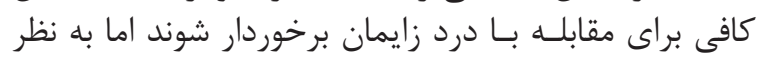

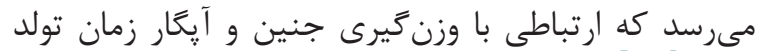

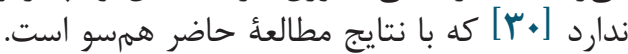

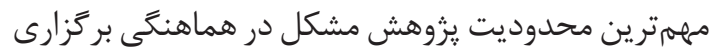

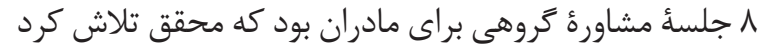

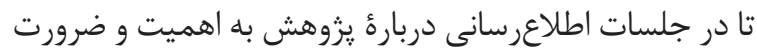

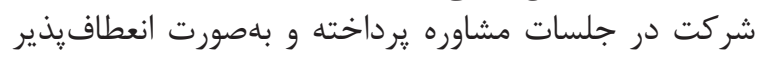

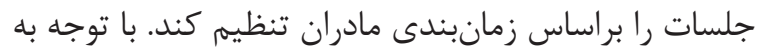

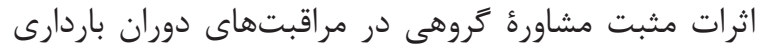

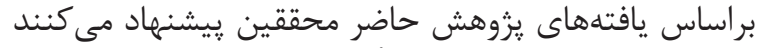

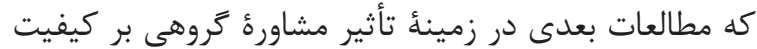
مراقبتهاى دوران باردارى انجام شود.

\section{نتيجه تيرى}

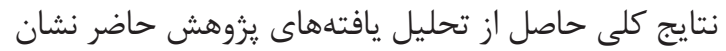

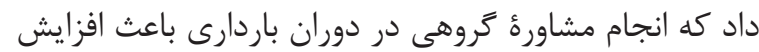

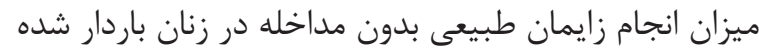

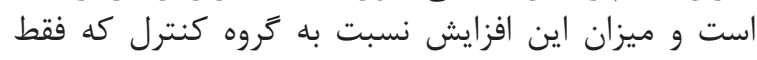

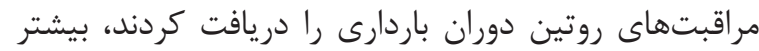

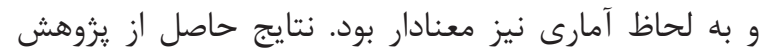

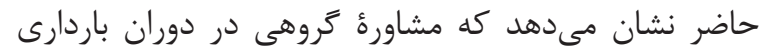

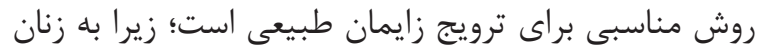

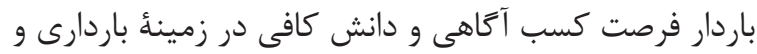

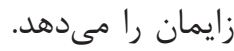

\section{سياسگزارى}

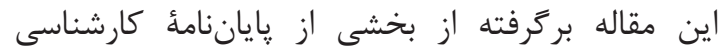

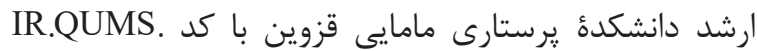

تحث

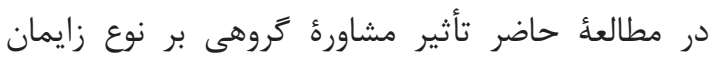

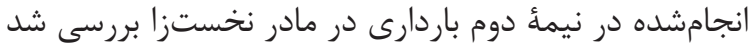

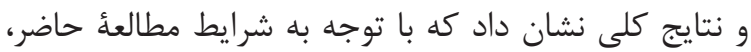

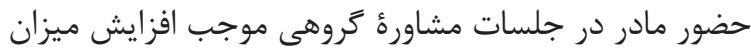

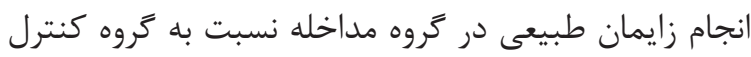

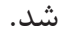

در مطالعئdajafi و همكاران (19 • (؟) نيز ميزان انتخاب

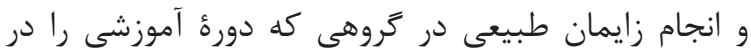

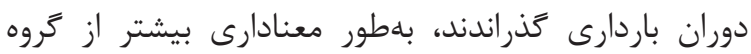

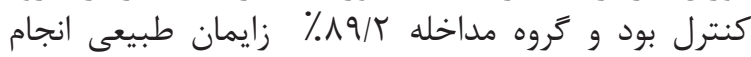

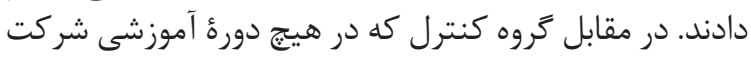

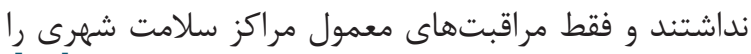

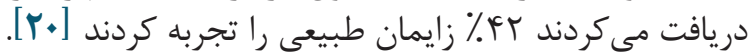
همجنين در مطالعه

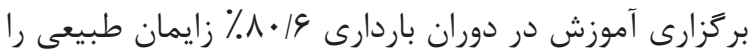

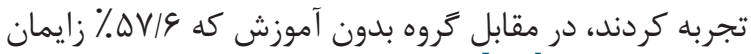

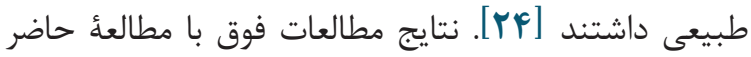

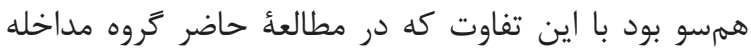

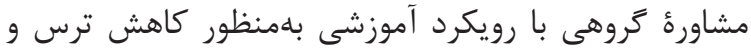

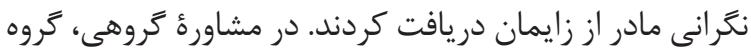

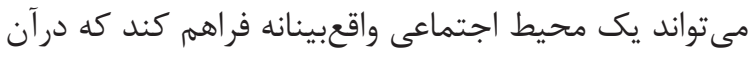

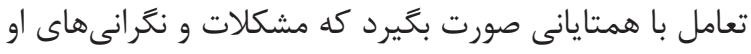

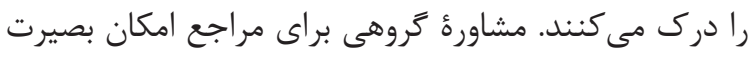

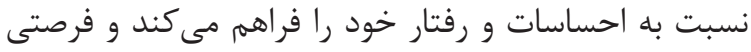

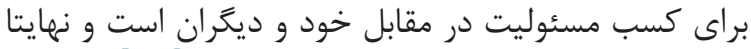

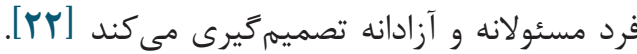

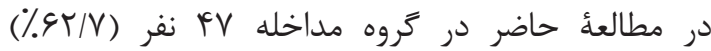

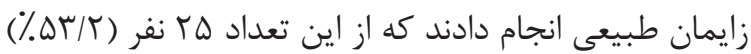

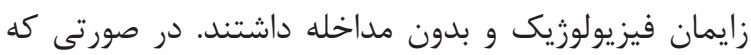

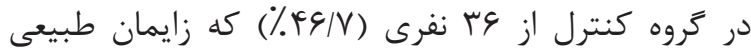

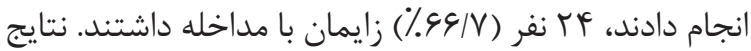

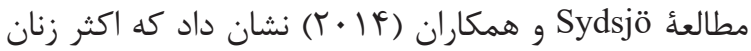

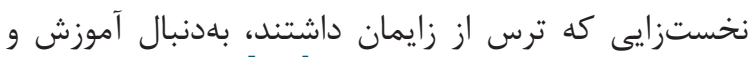

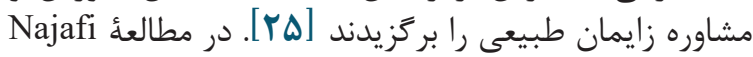

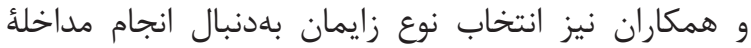

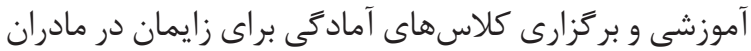

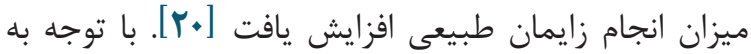

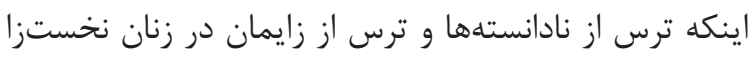

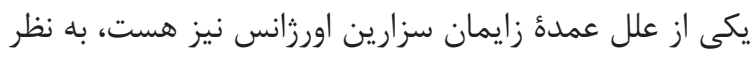

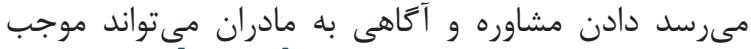

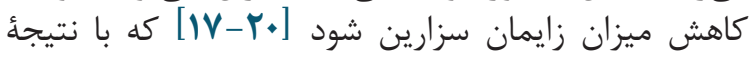
مطالعة حاضر همرسو است.

با توجه به نتايج مطالعه ميزان انجام زايمان طبيعى بروز

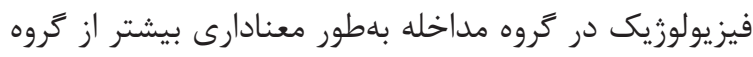

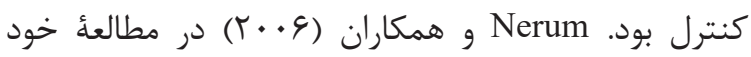

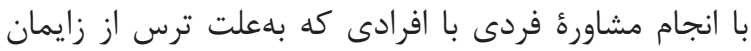
طبيعى متقاضى انجام سزارين انتخابى بودند، توانستند باديا مجله علمى دانشكده برستارى و مامايى همدان 


$$
\text { تمام مراحل انجام اين تحقيق يارى رساندند، تشكر و قدردانى }
$$

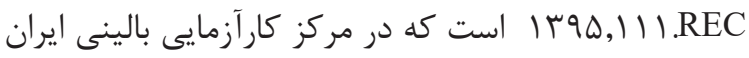

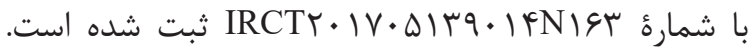

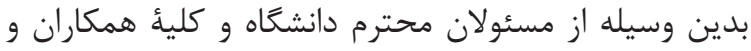

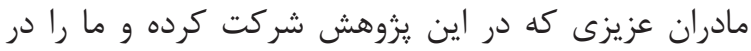

\section{References}

1. Abbasi Z, Abedian Z, Fadayi A, Esmaeil H. Effect of massage on physiologic responses on primiparous women. Horizon Med Sci. 2007;13(1):2833.

2. Cunningham F, Leveno K, Bloom S, Spong C, S.Dashe J. Williams Obstetrics. 24nd ed. McGraw-Hill Education; 2014.

3. Reza Soltani P, Hosseinjani A, Etebari S. Causes of cesarean section performed in an educational health care center. J Holist Nurs Midwifery. 2012;22(1):16-22.

4. Dadipoor S, Alavi A, Safari Moradabadi A. A survey of the growing trend of caesarian section in Iran and the world: a review article. Iran J Obstet Gynecol Infertil. 2016,19(27):8-17.

5. Ministry of Health, Treatment and Medical Education; 2012. Available from: www.behdasht. gov.ir /index.aspx?siteid=1\&pagei.

6. Melender H. Fears and coping strategies associated with pregnancy and childbirth in Finland. J Midwifery Womens Health. 2002;47(4):256-63. https://doi.org/10.1016/S1526-9523(02)00263-5

7. Sharifirad R, Fathian Z, Tirani M, Mahaki B. Study on Behavioral Intention Model (BIM) to the attitude of pregnant women toward normal delivery and cesarean section in province of Esfahan -Khomeiny shahr-1385. J Ilam Univ Med Sci. 2007;15(1):19-23. http://sjimu.medilam. ac.ir/article-1-15-en.html

8. Guszkowska M. The effect of exercise and childbirth classes on fear of childbirth and locus of labor pain control. Anxiety Stress Coping, 2013;27(2):176-89. https://doi.org/10.1080/106 15806.2013.830107 PMID:24199962

9. Nataša Jokić-Begić, Lana Žigić, Sandra Nakić Radoš. Anxiety and anxiety sensitivity as predictors of fear of childbirth: different patterns for nulliparous and parous women. J Psychosom Obstet Gynaecol. 2014;35(1):22-8. https://doi.org/10.3 109/0167482X.2013.866647 PMID:24328559

10. Toosi M, Akbarzadeh M, Zare N, Sharif F. The Role of Relaxation Training to Pregnant Mothers on Health Index of Infants. J Jahrom Univ Med Sci. 2013;11(1):15-21. https://doi.org/10.29252/ jmj.11.1.3

11. Shafiabadi A. Vocational and educational guidance and counseling (concepts and applications). Tehran: Samt; 2005.
12. Faghihi A. Consultation in the mirror of science and religion: Qom: Islamic Publications Office; 2005. PMCID:PMC550646

13. Nosratabadi M. Comparison of the effects of group and individual health counseling on knowledge, attitude and choice of delivery mode in first pregnant women [dissertation]. Ahvaz Iran: Ahvaz Jundishapur University of Medical Sciences; 2016.

14. Masoumi SZ, Kazemi F, Oshvandi K, Jalali M, Esmaeili-Vardanjani A, Rafiei H. Effect of training preparation for childbirth on fear of normal vaginal delivery and choosing the Type of delivery among pregnant women in Hamadan, Iran: A Randomized Controlled Trial. J Family Reprod Health. 2016;10(3):115-24. PMID:28101112 PMCID:PMC5241355

15. Kızılırmak A, Başer M. The effect of education given to primigravida women on fear of childbirth. Appl Nurs Res. 2016;29:19-24. https://doi.org/10.1016/j.apnr.2015.04.002 PMID:26856483

16. Delaram M, Soltanpour F. The Effect of Counseling in Third Trimester on Anxiety of Nulliparous Women at the Time of Admission for Labor. Zahedan J Res Med Sc. 2012;14(2):61-5. http:// zjrms.ir/article-1-1089-en.html

17. Taghizadeh z, Jafar Beyglou M, Arbabi M, Faghihzadeh S. The effect of counseling on posttraumatic stress disorder after a traumatic childbirth. Hayat. 2008;13(4):23-31. http://hayat.tums.ac.ir/ article-1-160-en.html

18. Azizi M, Lamyian M, Faghihzade S, Nematollahzade M. The effect of counseling on anxiety after traumatic childbirth in nulliparous women; a single blind randomized clinical trial. J Kermanshah Univ Med Sci. 2010;14(3):212-19.

19. Gamble J, Creedy D, Moyle W, Webster J, McAllister M, and Dickson P. Effectiveness of a counseling intervention after a traumatic childbirth: a randomized controlled trial. Birth. 2005;32(1):11-9. https://doi.org/10.1111/j.07307659.2005.00340.x PMID:15725200

20. Najafi F, Abouzari-Gazafroodi K, Jafarzadeh-Kenarsari F, Rahnama P, Gholami Chaboki B. Relationship between attendance at childbirth preparation classes and fear of childbirth and type of delivery. Hayat. 2016;21(4):30-40. http://hayat. tums.ac.ir/article-1-1257-en.html 
21. Ministry of Health and Medical Education (MoHME). Health Sector Evolution. Islamic Republic of Iran, Minstry of Health and Medical Education. Available from: http://tahavol.behdasht.gov.ir/. Accessed Feb 19, 2015

22. Gibson R, Mitchel M. Introduction to counseling \& guidance. New York: Macmillan; 1990.

23. Mental Health Department. Mother safe Country program: The integrated maternal health cares for midwife and General Practitioner. 4nd ed. Tehran: https://dawhois.com/www/enegah.com. html, 2016.

24. Tofighi Niaki M, Behmanesh F, Mashmuli F, Azimi H. The effect of prenatal group education on knowledge, attitude and selection of delivery type in primiparous women. Iran J Med Educ. 2010;10(2):124-30. http://ijme.mui.ac.ir/article1-1225-en.html

25. Sydsjö G, Bladh M, Lilliecreutz C, Persson A, Vyöni h, Josefsson A. Obstetric outcomes for nulliparous women who received routine individualized treatment for severe fear of childbirth-a retrospective case control study. BMC Pregnancy Childbirth. 2014;14:12641. https://doi.org/10.1186/1471-2393-14-126 PMID:24694283 PMCID:PMC4234140

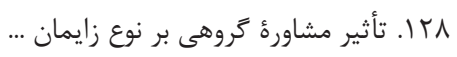

26. Nerum H, Halvorsen L, Sørlie T, Øian P. Maternal Request for Cesarean Section due to Fear of Birth: Can It Be Changed Through Crisis-Oriented Counseling? Birth. 2006;33(3):221-8. https:// doi.org/10.1111/j.1523-536X.2006.00107.x PMID:16948722

27. Melender HL. Fears and coping strategies associated with pregnancy and childbirth in Finland. J Midwifery Womens Health. 2002;47(4):256-63. https://doi.org/10.1016/S1526-9523(02)00263-5

28. Shariat M, Majlesi F, Azari S, Mahmoodi M. Caesarean section in maternity hospitals in Tehran, Iran. Payesh. 2002;1(3):5-10. http://www. sid.ir/En/Journal/ViewPaper.aspx?ID=45352

29. Delaram M, Raeisi Dehkordi SAR. The effect of counseling with nulliparous women during third trimester in pregnancy outcomes. Jundishapur Sci Med J. 2012;11(4):355-63.

30. Mehdizadeh A, Roosta F, Chaichian S, Alaghehbandan R. Evaluation of the impact of birth preparation courses on the health of the mother and the newborn. Am J Perinatol. 2005;22(1):79. https://doi.org/10.1055/s-2004-837738 PMID: 15668838 